\title{
BMJ Open The Oxford Renal (OxRen) cross-sectional study of chronic kidney disease in the UK
}

\author{
Nathan R Hill, ${ }^{1}$ Daniel Lasserson, ${ }^{1}$ Samuel Fatoba, ${ }^{1}$ Chris A O'Callaghan, ${ }^{2}$ \\ Chris Pugh, ${ }^{2}$ Rafael Perera-Salazar, ${ }^{1}$ Brian Shine, ${ }^{3}$ Ben Thompson, ${ }^{1}$ \\ Jane Wolstenholme, ${ }^{4}$ Richard McManus, ${ }^{1}$ F D Richard Hobbs ${ }^{1}$
}

To cite: Hill NR, Lasserson D, Fatoba S, et al. The Oxford Renal (OxRen) cross-sectional study of chronic kidney disease in the UK. BMJ Open 2013;3: e004265. doi:10.1136/ bmjopen-2013-004265

- Prepublication history and additional material for this paper is available online. To view these files please visit the journal online (http://dx.doi.org/10.1136/ bmjopen-2013-004265).

Received 16 October 2013 Accepted 4 November 2013

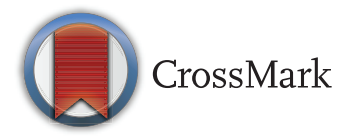

For numbered affiliations see end of article.

Correspondence to Professor Richard Hobbs; Richard.Hobbs@phc.ox.ac.uk

\section{ABSTRACT}

Introduction: Chronic kidney disease (CKD) diagnosed with objective measures of kidney damage and function has been recognised as a major public health burden. Independent of age, sex, ethnicity and comorbidity, strong associations exist between cardiovascular disease, mortality, morbidity and CKD, defined by reduced glomerular filtration rate and increased urinary albumin excretion. Detection of CKD within the population is therefore a priority for health systems.

Methods and analysis: 15000 patients aged 60 years or over meeting the inclusion criteria will be invited to the study. Recruitment will be stratified to represent the distribution of socioeconomic position in the UK general population. Patients will be excluded if terminally ill (expected survival $<1$ year), or if they have received a solid organ transplant. Patients will attend up to two screening visits, to determine if they have CKD, followed by an assessment visit where demographic and physiological parameters will be recorded alongside questionnaires on exercise, diet, cognitive assessment and quality of life. Blood and urine specimens will be taken for immediate routine assays as well as for freezing pending peptide and genetic studies. Patients will have office and home blood pressure measurements as well as pulse wave velocity assessment. Healthcare costs of screening and subsequent monitoring will be calculated.

Ethics and dissemination: The protocol and related documents have been approved by NRES Committee South Central-Oxford B-Reference 13/SC/0020.

\section{INTRODUCTION}

Chronic kidney disease (CKD) is a worldwide health problem associated with high morbidity and mortality ${ }^{12}$ and its prevalence is increasing. ${ }^{3}$ Decreased renal function is a well-known predictor of hospitalisation, ${ }^{4}$ cognitive dysfunction $^{5}$ and reduced quality of life (QoL). ${ }^{6} 7$ Cardiovascular disease (CVD) is the primary cause of morbidity and mortality in this population where CKD is regarded as an accelerator of CVD risk and an independent risk factor for CVD events. ${ }^{4}$ Even the earliest stages of
CKD are known to be associated with significantly increased risks of cardiovascular morbidity, premature mortality and decreased QoL. ${ }^{4} \quad 8$ While the CVD risk in end-stage renal failure is high, the healthcare burden resides in early stages of disease as it is more prevalent, affecting around $35 \%$ of those over 70 years. $^{9}$

Interventions for patients with CKD can delay progression, decrease morbidity ${ }^{10}$ and decrease mortality rates. ${ }^{11}$ Antihypertensive medications blocking the renin-angiotensin-aldosterone system-such as ACE inhibitors and/or angiotensin receptor blockers-have been systematically proven to be of benefit to patients with proteinuric CKD. ${ }^{12}$ A recent systematic review by Sharma reported a statistically significant reduction in the risk of doubling of creatinine levels (in people with stage 3 CKD stratified by baseline $24 \mathrm{~h}$ urinary protein) in those with $\geq 3 \mathrm{~g} / \mathrm{L}$ of proteinuria $(66 \%, 95 \%$ CI $34 \%$ to $82 \%)$. Numerous other studies examining the role of interventions have also been shown to be effective in CKD to delay progression and/ or improve mortality rates, including statin therapy, ${ }^{13}$ blood pressure (BP) lowering, ${ }^{14} 15$ control of metabolic bone disease ${ }^{16}$ and lifestyle changes in diet ${ }^{17}$ and physical activity. ${ }^{18}$

Early stages of CKD are defined by the combination of kidney damage (quantified with evidence of renal damage-imaging or proteinuria) and decreased kidney function (defined as glomerular filtration rate (GFR) estimated from serum creatinine concentration). The National Institute for Care Excellence $(\mathrm{NICE})^{19}$ recommends using serum creatinine concentration for estimation of GFR (eGFR) using the Modification of Diet in Renal Disease study four-variable equation. ${ }^{20}$ The NICE highlights the need for strategies aimed at earlier identification and (where possible) prevention of progression to established renal failure. ${ }^{19}$ Since CKD is usually asymptomatic until later stages of the disease (stage $4+$ ), it may be beneficial to 
establish efficient detection mechanisms for patients with early-stage CKD (figure 1). ${ }^{21}$

Screening enables early detection, but mass screening of all age groups for kidney disease is expensive, of low yield and not cost-effective. ${ }^{22}$ There is an age-related decline in renal function, and it has been shown in contemporary UK data sources ${ }^{9}{ }^{23}$-including retrospective laboratory data, audit studies and general practitioner (GP) record systems-that from age 60 years, CKD prevalence increases rapidly. It may, therefore, be reasonable to screen systematically in this age group.

The primary aim of this study is to establish the number of previously undetected cases of CKD. They will be detected by screening of a blood test for the serum creatinine result and calculation of the eGFR and a quantification of urinary protein.

The secondary aims are to (1) determine the costeffectiveness of screening for CKD and (2) determine the prevalence of selected risk factors and levels of distribution of estimated kidney function in screen-detected patients.

\section{METHODS AND ANALYSIS \\ Study design}

This cross-sectional screening study is part of the Oxford Renal Study (OxRen), recruiting from a population of patients aged 60 years and older who are registered at GP surgeries within the Thames Valley region. OxRen consists of a further study that will longitudinally follow-up patients who are diagnosed with CKD through this screening study and elsewhere.

The Thames Valley has 278 practices and supports a population of approximately 2.3 million. OxRen consists of a co-ordinating centre, the NIHR School for Primary Care Research (SPCR) Oxford and a minimum of 11 GP surgeries (covering a population of approximately 60000 patients) from which patients are recruited. There will be up to three additional practices that will serve as a control group for the cost-effectiveness analysis.

\section{Inclusion criteria}

- Participant is willing and able to give informed consent for participation in the study.

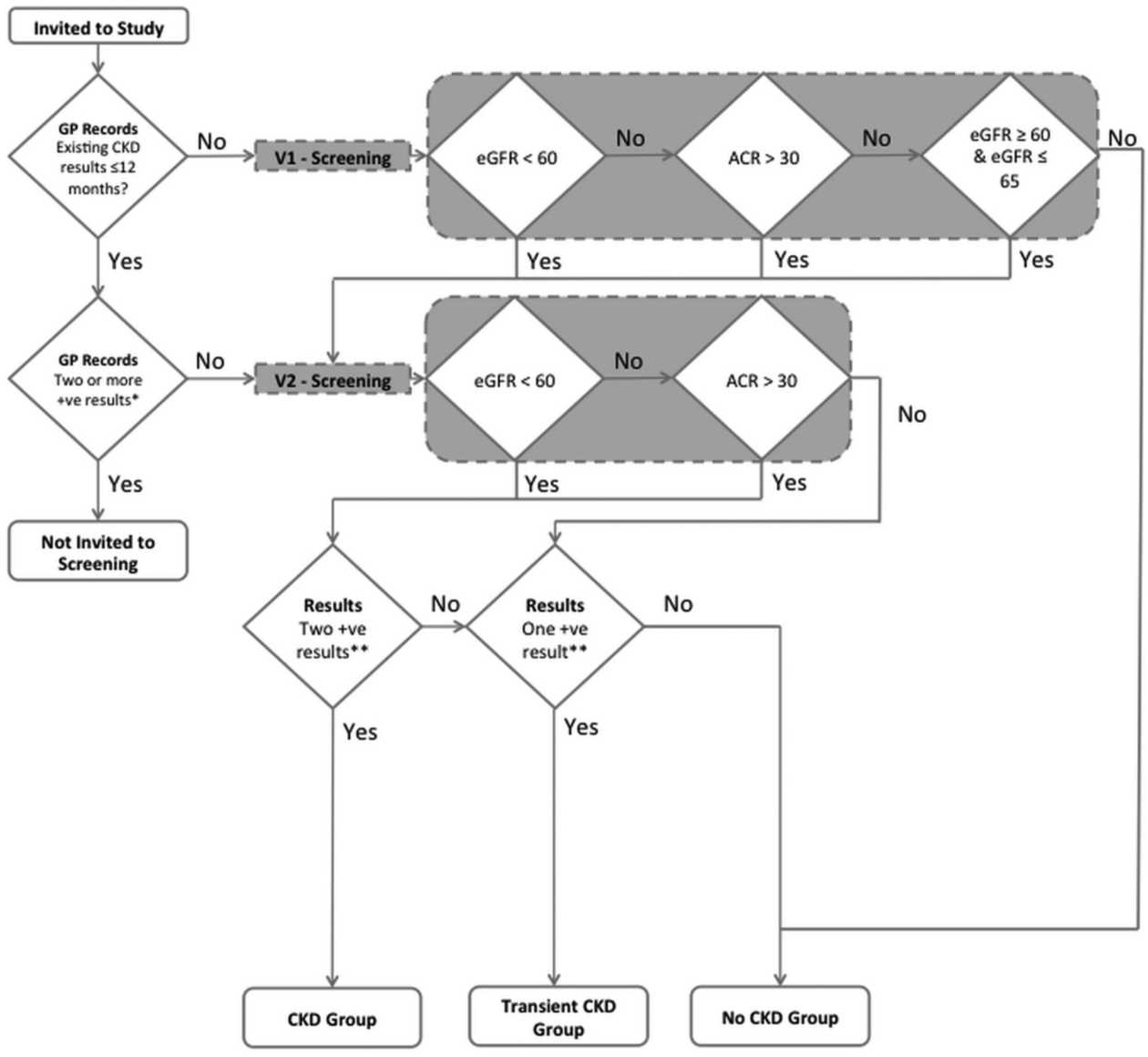

Figure 1 Patient flow diagram. *NICE criteria for diagnosis of CKD-two or more results at least 3 months apart in the past 12 months. ${ }^{* *}\left(\right.$ eGFR $<60 \mathrm{~mL} / \mathrm{min} / 1.73 \mathrm{~m}^{2}$ ) or (eGFR $>60 \mathrm{~mL} / \mathrm{min} / 1.73 \mathrm{~m}^{2}$ and $A C R>30 \mathrm{mg} / \mathrm{mmol}$ ) at least one result must be from V1 or V2. ACR, albumin creatinine ratio; CKD, chronic kidney disease; eGFR, estimated glomerular filtration rate; NICE, National Institute for Care Excellence. 
- Men or women, aged 60 years or above.

- No blood test within the past 12 months.

\section{Exclusion criteria}

In order to be as inclusive as possible there are a minimal number of exclusion criteria:

- Not previously diagnosed with CKD (either coded by their GP or someone else and any recorded eGFR $>90 \mathrm{~mL} / \mathrm{min} / 1.73 \mathrm{~m}^{2}$ within the past 12 months or other renal disease);

- Terminally ill (expected survival $<1$ year);

- Previous solid organ transplant;

- Patients whom the GP feel are inappropriate for the study;

\section{Recruitment process}

Patients will be identified by staff, at GP surgeries, who will review the electronic patient records and select patients who fulfil the study criteria.

A spread of practices representative of the UK general population will be achieved by stratifying practices into national quartiles of deprivation, taking into account practice size and selecting practices that agree to take part sequentially until each quartile practice target is reached. Over the course of recruitment, the sequential practice selection strategy will be examined to ensure practices serving high proportions of ethnic minorities are included in the final places if this has not already occurred in earlier recruitment.

\section{Study patients}

Fifteen thousand patients aged over 60 years from a primary care population will be invited by letter to be part of the study. The letter will include the study patient information sheet. Patients will be given a contact number and email address of the study team who will be able to answer any questions they might have.

Patients' first visits will depend on any previous record of impaired renal function as defined by an abnormal eGFR (eGFR $\geq 60 \mathrm{~mL} / \mathrm{min} / 1.73 \mathrm{~m}^{2}$ and albumin to creatinine ratio (ACR) $\geq 30 \mathrm{mg} / \mathrm{g}$ or eGFR $<59 \mathrm{~mL} / \mathrm{min} /$ $1.73 \mathrm{~m}^{2}$ regardless of ACR).

No prior record of abnormal eGFR: Patients with no prior record of impaired renal function will attend the first screening visit (visit 1) for a blood and urine test to determine CKD status. If at this visit they are deemed to have an impaired renal function they will then proceed to a second screening visit (visit 2) after a 3-month interval to have their CKD diagnosis confirmed. The results must be at least 3 months apart and within the past 12 months to be in line with the NICE guidance.

Participants within $5 \mathrm{~mL} / \mathrm{min} / 1.73 \mathrm{~m}^{2}$ of the upper limit eGFR range of stage 3a CKD (ie, between 60 and $65 \mathrm{~mL}$ / $\min / 1.73 \mathrm{~m}^{2}$ ) will be invited to the second screening visit (visit 2). This is to ensure the intraindividual variation in eGFR does not lead to a false-negative classification of disease. Patients will also undergo a urinary ACR.
One positive result for $C K D$ : Patients with a previously recorded single result for impaired renal function (within the past 12 months), defined by an abnormal eGFR and/ or urinary protein, will have CKD status determined with a confirmatory blood test for calculation of eGFR as well as urinary ACR. If CKD is diagnosed in participants then they will attend the baseline assessment (visit 3).

All patients diagnosed with CKD through at least two abnormal eGFRs and/or ACRs will attend the baseline assessment visit (visit 3). If the patient's eGFR and/or ACR tests are abnormal at visit 1 and normal at visit 2 they will be defined as having transient CKD and will proceed to the baseline assessment visit (visit 3). All other patients will not attend the baseline assessment visit.

\section{CKD definition}

CKD diagnoses will be established through eGFR and evidence of renal damage (proteinuria-ACR) to define the early stages. The eGFR will be estimated using the MDRD equation using an Isotope-Dilution Mass Spectrometry (IDMS) compatible creatinine assay as recommended by NICE. The CKD-EPI equation for estimation of eGFR will be used as a method to determine whether there is any difference in CKD categorisation as the MDRD may underestimate the actual GFR in healthy patients by up to 29\%. ${ }^{24}$ Albuminuria is defined using ACR with microalbuminuria being an ACR of $3.4(30 \mathrm{mg} / \mathrm{g})$ to $33.9 \mathrm{mg} / \mathrm{mmol}$ $(299 \mathrm{mg} / \mathrm{g})$. Sex-specific cut-offs for ACR will also be used $^{25}$ : microalbuminuria $(1.9 \mathrm{mg} / \mathrm{mmol}(\geq 17 \mu \mathrm{g} / \mathrm{mg})$ in men and $2.8 \mathrm{mg} / \mathrm{mmol}(\geq 25 \mu \mathrm{g} / \mathrm{mg})$ in women $)$. Macroalbuminuria will be defined as an ACR $\geq 34 \mathrm{mg}$ / $\mathrm{mmol}(300 \mathrm{mg} / \mathrm{g})$. Stages of CKD will be defined in line with the NICE guidelines as shown in table 1.

\section{Outcome measures}

Primary outcome: The proportion of patients diagnosed with CKD from a primary care population of patients aged $60+$ years demographically representative of the UK general population.

Secondary outcome: (1) The cost and cost-effectiveness of screening for CKD in the 60+ age group and (2) the prevalence of selected risk factors and the distribution of eGFRs within that group.

\begin{tabular}{lll}
\multicolumn{4}{l}{ Table 1} & CKD stages defined by eGFR and ACR \\
\hline CKD stages & eGFR $\left(\mathbf{m L} / \mathbf{m i n} / \mathbf{1 . 7 3} \mathbf{~ m}^{\mathbf{2}}\right)$ & ACR $\mathbf{( m g / g )}$ \\
\hline 1 & $\geq 90$ & $\geq 30$ \\
2 & $60-89$ & $\geq 30$ \\
3a & $45-59$ & - \\
3b & $30-44$ & - \\
4 & $15-29$ & - \\
5 & $<15$ & - \\
\hline & ACR, albumin creatinine ratio; CKD, chronic kidney disease; \\
eGFR, estimated glomerular filtration rate.
\end{tabular}




\section{Study procedures}

Patient visits are at their own GP practice. Practitioners will be trained in study procedures including obtaining informed consent. The study will be carried out in accordance with the Declaration of Helsinki and OxRen. An algorithm, that specifies what nephrology advice is given should a patient be diagnosed with CKD, will be provided to GPs. Patients who are identified for the first time as having advanced CKD (eGFR $<30 \mathrm{~mL} / 1.73 \mathrm{~m}^{2}$, stage $4+$ ) will be referred to a specialist renal clinic.

\section{Screening visit (visit 1)}

The study will be explained to potential participants and they have the opportunity to ask questions about the study. Consent will be sought and the potential participants have as much time as required to decide whether they wish to participate. If they consent, patients will have a blood test for calculation of kidney function using the MDRD eGFR and urinary assessment for ACR.

Patients who test positive will proceed to visit 2-as will patients within $5 \mathrm{~mL} / \mathrm{min} / 1.73 \mathrm{~m}^{2}$ of the upper limit eGFR range of stage 3a CKD (ie, between 60 and $65 \mathrm{~mL}$ / $\min / 1.73 \mathrm{~m}^{2}$ ). Patients who test negative will not proceed.

\section{Screening visit (visit 2)}

Patients with a single eGFR and/or ACR in the range for a CKD diagnosis will have a second confirmatory blood test for kidney function performed at this visit. If after this visit the patient has two abnormal results for impaired renal function, they will be classified as being diagnosed with CKD and invited to attend the baseline assessment (visit 3). Patients with an eGFR $>60 \mathrm{~mL} / \mathrm{min} / 1.73 \mathrm{~m}^{2}$ but persistent proteinuria as evidenced by a raised ACR at visit 2 will also be diagnosed and invited to attend the baseline assessment.

If on the second occasion the patient's blood results do not fall within the criteria for CKD diagnosis, patients will still be invited to the assessment baseline visit (visit 3) but classified as having 'transient CKD' and will proceed to the next visit (visit 3-baseline assessment).

\section{Baseline assessment visit (visit 3)}

The baseline assessment visit will occur after the screening visits (visits 1 and 2). Patients have full demographic details recorded including age, self-assigned ethnicity, educational status, residential postcode, clinical history, medical history, family history, smoking and alcohol use, major comorbidities, current medication, physical examination (weight, height and waist circumference using a validated method) and BP measurement using a validated automated device. Patients will be submitted to a 12-lead ECG.

Blood specimens will be obtained for creatinine and electrolytes, blood glucose, hepatic and bone profiles, glycated haemoglobin (HbAlc), lipids (total cholesterol, low-density lipoprotein, high-density lipoprotein), urate, B-type natriuretic peptide, cystatin C, full blood count and a random spot urine sample for the calculation of the ACR. Serum creatinine will be measured by automatic analyser (using the Jaffe method calibrated to IDMS values). Five sample bottles $(20 \mathrm{~mL} / \mathrm{s}$ in total) will be required to collect the blood specimens for analysis by the biochemistry laboratory. One $20 \mathrm{~mL}$ sample bottle will be required for the collection of urine. All samples will be sent to the laboratory from the GP practices in the routine way.

Additional samples ( 5 bottles, $25 \mathrm{mLs}$ ) will be collected and stored at $-80^{\circ} \mathrm{C}$ for future genetic and protein testing. One additional $20 \mathrm{~mL}$ sample bottle for urine will be collected. All samples will be sent to the laboratory with the blood specimens for the routine haematology and biochemistry. Once at the laboratory they will be prepared and sent on to the bio-bank.

Patients will be given questionnaires to be completed at visit 3 , taking approximately $50 \mathrm{~min}$ to complete.

- QoL: assessed using the EQ-5D-5 L questionnaire-six items taking approximately $5 \mathrm{~min}$ to complete.

- Dietary assessment: assessed using the Million Woman Study Diet Questionnaire-nine items taking approximately 15 min to complete.

- Physical activity: assessed using the International Physical Activity Questionnaire: Short version (iPAQ-Short)seven items taking approximately $10 \mathrm{~min}$ to complete.

- Cognitive function: assessed using the Montreal Cognitive Assessment questionnaire-eight tasks requiring approximately $20 \mathrm{~min}$ to complete.

Patients are provided with a BP monitor suitable for home use and asked to document their BP three times daily over a typical week and to return it. We also seek to provide patients with a $24 \mathrm{~h}$ BP monitor. The device will be provided following the baseline visit for use on 1 day in a typical week and will then be returned. Further BP information will be collected using a pulse wave analysis machine that will be used on patients at their clinic visits.

Hypertension will be defined as a clinical measurement of systolic BP of $140 \mathrm{~mm} \mathrm{Hg}$ or greater, diastolic BP $90 \mathrm{~mm} \mathrm{Hg}$ or greater confirmed with ambulatory BP monitoring ${ }^{26}$ or use of antihypertensive medications irrespective of BP. Diabetes mellitus will be defined as HbAlc of $48 \mathrm{mmol} / \mathrm{mol}(6.5 \%)$ or greater or use of hypoglycaemic agents. ${ }^{27} \mathrm{~A}$ history of CVD is defined as a history of coronary arterial disease, cerebrovascular disease or peripheral arterial disease. Body mass index will be calculated based on weight and height (weight $(\mathrm{kg}) /$ height $\left(\mathrm{m}^{2}\right)$ ). Any new diagnosis of disease will be followed up with patients' GP informing them of the NICE guidelines in the relevant area.

\section{Procedures}

Oxford University Hospitals NHS Trust Clinical Biochemistry laboratories will perform all the tests using standardised methods that are regularly recalibrated against reference samples.

\section{Number of patients}

Fifteen thousand patients will be invited to the study. We estimate a recruitment rate of $40 \%$ per practice giving a 
cohort of 6000 patients. We require a total practice population of 82500 (or about 11 GP surgeries) determined from an average practice size with a patient list of 7500. This is based on data from the Oxford Vascular Study of 4627 people (January 2012) within Oxfordshire, that detailed the percentage of an average practice list that is over 60 is $21 \% .^{28}$

\section{Analyses}

To estimate the benefits of screening for the diagnosis of CKD using a blood test for the eGFR, we have based the calculation on the cohort of 6000 patients. Prevalence estimates for CKD vary between $20 \%{ }^{23}$ and $28 \%{ }^{9}$ for patients who are $\geq 60$ years. Using a sample size of 6000 and a $20 \%$ and $28 \%$, as lower and upper limits for the estimated prevalence, this number of patients with CKD will yield a precision (defined as the total length of $95 \% \mathrm{CI}$ ) between $1 \%$ and $1.1 \%$ around the prevalence estimate.

Descriptive analyses will be used to characterise the participant population by sociodemographic data, health status and lifestyle factors. The prevalence and mean values of CKD stages and selected conditions (diabetes, hypertension, etc) by sex, age and race/ethnicity will be examined using $\chi^{2}$ statistics for categorical variables and Wilcoxon's rank sum for continuous values. We will carry out univariate analyses to explore the associations between different patient characteristics and eGFR levels as a continuous variable at baseline and we will explore multivariate models that will include these characteristics such as age, gender and the other factors we measure during the baseline assessment.

\section{Economic analysis}

The health economic analysis will consider three questions.

- What is the cost to the National Health Service (NHS) of screening for CKD, in terms of the cost of the screen and subsequent healthcare costs related to treating and managing the disease?

- How much cost-effective is the systematic screendetection compared with the current detection of CKD via the provision of routine care, in terms of cost per additional case detected?

- What is the long-term cost-effectiveness of screen detection/active case finding in terms of cost per additional quality-adjusted life year (QALY) gained?

For the purpose of the economic analysis, the cohort of individuals invited to be screened will need to be compared with a control cohort with no screening. It is proposed that up to three practices will be recruited to act as a control group. The economic evaluation will be split into two components. The cost per case detected will be estimated using the data collected from individuals in the cohort. The long-term cost-effectiveness of screening/case-finding will be determined by developing a life-time disease model for CKD.
Data collection

Resource use and costs: The cost analysis will adopt an NHS perspective.

Screening/case finding cost: The cost of screening/case finding will be determined using data from the invited cohort of individuals. Resource use and unit costs will be collected in relation to the identification of the cohort through routine clinical records, the invitation and diagnostic tests performed.

Cost related to follow-up healthcare. Data on healthcare resource use will be collected from all individuals in the 'invited-to-screen' and 'no-screen' cohorts, including all relevant hospital and GP consultations, medications, referrals, tests and equipment. Where possible, data on resource utilisation will be collected from the electronic patient records. One-third of the records for this cohort will be amenable to this form of data collection. For the remainder of the cohort we will set up an electronic data-capture for a number of predetermined healthcare resource-use events which are likely to represent key cost-drivers, such as medications and hospitalisations.

Where possible, we will value our items of healthcare resource utilisation using appropriate unit costs (staff costs, equipment, drug costs, etc) obtained from the published sources, including the most recent version of Unit Costs of Health and Social Care and NHS Reference Costs. We will estimate unit costs that are not available from secondary sources using the approach used in the most recent version of Unit Costs of Health and Social Care.

Outcomes/utility data: The outcome of interest for the purpose of the economic evaluation will be the number of CKD cases detected/diagnosed. This will be obtained directly from the screen-detected cohort and compared with the numbers arising in the 'no-screen' control group.

The NICE recommends the use of preference-based health-related quality of life measures for the purpose of determining QALYs for economic evaluation. The use of QALYs aims to capture the impact of disease progression and non-fatal events on QoL in addition to any impact on survival. The EQ-5D will be used to measure patient health-related QoL across five-dimensions (5D), mobility, self-care, usual activities, pain/discomfort, anxiety and depression. It will be collected from the cohort at baseline and at their annual follow-up contact. EQ-5D scores at each time point will be converted into a utility score on a $0-1$ scale where 0 is equivalent to dead and 1 to perfect health. This conversion will be made using the new algorithm based on the UK value set currently being conducted by the EuroQol Group, if available at the time of analysis. If not available, the current crosswalk algorithm provided by the EuroQol group and algorithm estimated by Dolan $e t$ al derived from a survey of the UK population $(n=3337)$ will be used. Utility values in the tariff set range from no problems on any of the $5 \mathrm{D}$ in the EQ-5D descriptive system (value $=1.0$ ) to severe or extreme problems across all 5D (value $=-0.594) .{ }^{29} 30$ 
The utility scores will be combined with survival data to estimate the QALYs required for the cost-utility analysis. This utility data will be used for the purpose of modelling the cost per QALY of screening for CKD.

\section{Analysis}

Missing data: The resource-use/cost and EQ-5D data will be investigated to ascertain the extent of missing data and whether this is due to random missingness and/or censoring. Standard methods will be used to handle any relevant missing data. ${ }^{31} 32$

Cost analysis: The focus of studying the healthcare resource use is to investigate how screening for CKD affects the healthcare costs of treating and managing the disease. With the aim of the economic analysis to estimate how the costs of the 'screen/case-finding' minus the difference in healthcare costs between the screen-detected and routinely diagnosed group of patients balances against the healthcare benefits. A three-stage analysis of the healthcare resource use and their costs will be conducted. First, the cost associated with the case-finding/screen will be estimated using the resource-use related to the identification of the cohort through routine clinical records, the invitation and diagnostic tests performed (including staff time, equipment, tests and consumables). Second, the impact of the screendetection on (1) all healthcare resource use/costs, (2) kidney disease-specific healthcare resource use/costs and (3) CVD-related healthcare resources costs will be evaluated over the duration of the study (3 years). Third, a regression framework that relates healthcare costs to baseline characteristics age, gender, kidney disease stage, progression, other comorbidities and CVD will be developed. The objective to provide estimates of healthcare costs for different stages of kidney disease and CVD events which will be used to inform the extrapolation model (see below). A similar regression framework approach will be used for the EQ-5D tariff data at the different data collection timepoints, again to inform the extrapolation model.

Cost per-CKD case detected: The incremental costeffectiveness of screening compared with routine diagnosis of CKD will be determined using the cost analysis data (described above); cost of screening plus its cost impact on treating and managing CKD in the 'screen-detected' cohort will be compared with healthcare costs related to CKD in the routine diagnosis cohort. This incremental cost will be weighed up against the incremental benefit in terms of the incremental CKD cases detected by screening compared with routine diagnosis over the initial 2-year period that the entire screened cohort will run. Discounting at a rate of $3.5 \%$ will be applied. By using data from the cohort that uses Thames Valley practices only, there may be limits to the generalisability of the results of the study. This will be explored within the economic evaluation using extensive sensitivity analysis. The key parameters will be varied to determine the impact of changes on results. Case finding in different patient subgroups will also be considered. Non-parametric bootstrapping and probabilistic sensitivity analysis will be undertaken to explore the uncertainty in the confidence to be placed on the results of the economic analysis and cost-effectiveness acceptability curves will be presented.

Cost per QALY of screen-detection in CKD: Building on the results of the cohort-based economic evaluation (incremental cost-per-case detected), a model-based analysis will be conducted to estimate the cost per QALY of screening for CKD. The methods used will depend on the available cohort data, but will either use parametric methods as set out by the NICE Decision Support Unit ${ }^{33}$ or use a lifetime decision-model (developing a Markov model similar to that used by Manns $e t a t^{22}$ ) or adapting available CKD models. This will be based on the individual patient data (using the results from the regression analyses outlined above) from the study and external data (where required). It will be carried out from an NHS and Personal Social Services perspective, to take into account the healthcare costs and longer term social care costs of cardiovascular events and the impact on life expectancy and quality-adjusted life expectancy. The model will be run over remaining patient lifetime, with costs and benefits discounted at a rate of $3.5 \%$. The lifetime cost-effectiveness analysis will be driven by the decision analytic model and the way treatment effects are propagated in the model. Extensive deterministic sensitivity analysis will be undertaken to assess the impact of changing the values of key parameters and will be used to explore the importance of modelling assumptions. Probabilistic sensitivity analyses will be conducted to deal with the uncertainty in model parameters and costacceptability curves will be presented.

\section{ETHICS AND DISSEMINATION \\ Ethics}

The chief investigator will ensure that this study is conducted in accordance with the principles of the Declaration of Helsinki and ensure this study is conducted in full conformity with relevant regulations and with the ICH Guidelines for Good Clinical Practice (CPMP/ICH/135/95) July 1996. Site staff will be fully trained in Good Clinical Practice (GCP) as appropriate to their study role.

The chief investigator will submit and, where necessary, obtain approval for all substantial amendments to the original approved documents.

Ensuring patient confidentiality is an established and robust process within the Primary Care Clinical Trials Unit (PC-CTU) where the study data will be run. All staff adheres to the principles of GCP and the Data Protection Act, 1998. It is the PC-CTU's preferred procedure that patients will only be identified on study documents by use of a unique screening identity (ID) and, if diagnosed with CKD, a unique participant ID, which cannot be used to identify individual participants. All study documents such as case report forms holding patient information are held securely with restricted access either electronically or in paper format. 
The holding of patient identifiable information will be kept separate from the information collected on the patient and will be used only to follow-up the patient; for example, if a member of the research team needs to make a follow-up phone call to a patient or recall them for their annual follow-up visit. Case report forms and all other documents holding identifiers are anonymised as soon as possible with the process of management being outlined in detail within the ethics application and in study-specific procedures.

\section{Dissemination}

The investigators will be involved in reviewing the drafts of the manuscripts, abstracts, press releases and any other publications arising from the study. The authors will acknowledge that the National Institute for Health Research Oxford Biomedical Research Centre (NIHR BRC) and the NIHR School of Primary Care Research funded the study. Authorship will be determined in accordance with the ICMJE guidelines and other contributors will be acknowledged.

The results of this study will be of relevance to the renal research community and the primary care research community. We would present our findings to both a primary care audience (eg, at scientific meetings of the Society for Academic Primary Care and the Confederation of Primary Care Research Organisations) and a renal audience (eg, at the Renal Association and American Society of Nephrology).

It is expected that this study will generate a highimpact manuscript. The findings of the study will be submitted for publication in an appropriate peer-reviewed medical journal. Where possible, we would seek to publish in open access journals to encourage the dissemination of the information.

\section{Author affiliations}

${ }^{1}$ Department of Primary Care Health Sciences, Radcliffe Observatory Quarter, Oxford, UK

${ }^{2}$ Henry Wellcome Building for Molecular Physiology, Nuffield Department of Clinical Medicine, Churchill Hospital, Oxford, UK

${ }^{3}$ Department of Clinical Biochemistry, Oxford Radcliffe Hospitals NHS Trust, John Radcliffe Hospital, Oxford, UK

${ }^{4}$ Department of Public Health, University of Oxford, Oxford, UK

Contributors RH, RM, DL, COC and NRH conceived and designed the study. $\mathrm{NRH}, \mathrm{BT}, \mathrm{SF}$ and $\mathrm{DL}$ are supervising all recruitment and enrolment. JW is providing support and interpretation for the health economics, RP-S supervised the statistical analysis, BS provided laboratory/pathology supervision and analysis, CP and COC provided nephrology expertise and oversight. All authors contributed substantially to the manuscript's revision. $\mathrm{RH}$ takes responsibility for the paper as a whole.

Funding The research was funded by the National Institute for Health Research Oxford Biomedical Research Centre (NIHR BRC) and the NIHR School of Primary Care Research. The views expressed are those of the author(s) and not necessarily those of the NHS, the NIHR or the Department of Health

Competing interests None.

Ethics approval NRES Committee South Central-0xford B-Reference 13/SC 0020.
Provenance and peer review Not commissioned; internally peer reviewed.

Open Access This is an Open Access article distributed in accordance with the Creative Commons Attribution Non Commercial (CC BY-NC 3.0) license, which permits others to distribute, remix, adapt, build upon this work noncommercially, and license their derivative works on different terms, provided the original work is properly cited and the use is non-commercial. See: http:// creativecommons.org/licenses/by-nc/3.0/

\section{REFERENCES}

1. Keith DS, Nichols GA, Gullion CM, et al. Longitudinal follow-up and outcomes among a population with chronic kidney disease in a large managed care organization. Arch Intern Med 2004;164:659-63.

2. Gansevoort RT, Correa-Rotter R, Hemmelgarn BR, et al. Chronic kidney disease and cardiovascular risk: epidemiology, mechanisms, and prevention. Lancet 2013;382:339-52.

3. Coresh J, Selvin E, Stevens LA, et al. Prevalence of chronic kidney disease in the United States. JAMA 2007;298:2038-47.

4. Go AS, Chertow GM, Fan D, et al. Chronic kidney disease and the risks of death, cardiovascular events, and hospitalization. $N$ Engl J Med 2004;351:1296-305.

5. Etgen $\mathrm{T}$, Chonchol M, Forstl $\mathrm{H}$, et al. Chronic kidney disease and cognitive impairment: a systematic review and meta-analysis. Am J Nephrol 2012:35:474-82.

6. Perlman RL, Finkelstein FO, Liu L, et al. Quality of life in chronic kidney disease (CKD): a cross-sectional analysis in the Renal Research Institute-CKD study. Am J Kidney Dis 2005;45:658-66.

7. Chin HJ, Song YR, Lee JJ, et al. Moderately decreased rena function negatively affects the health-related quality of life among the elderly Korean population: a population-based study. Nephrol Dial Transplant 2008;23:2810-17.

8. Chow FY, Briganti EM, Kerr PG, et al. Health-related quality of life in Australian adults with renal insufficiency: a population-based study. Am J Kidney Dis 2003;41:596-604.

9. O'Callaghan CA, Shine B, Lasserson DS. Chronic kidney disease: a large-scale population-based study of the effects of introducing the CKD-EPI formula for eGFR reporting. BMJ Open 2011;1: e000308.

10. Eknoyan G, Levey AS, Levin NW, et al. The national epidemic of chronic kidney disease. What we know and what we can do. Postgrad Med 2001;110:23-9: quiz 8.

11. Tonelli M, Wiebe N, Culleton B, et al. Chronic kidney disease and mortality risk: a systematic review. J Am Soc Nephrol 2006;17:2034-47.

12. Sharma P, Blackburn RC, Parke CL, et al. Angiotensin-converting enzyme inhibitors and angiotensin receptor blockers for adults with early (stage 1 to 3) non-diabetic chronic kidney disease. Cochrane Database Syst Rev 2011;(10):CD007751.

13. Baigent $\mathrm{C}$, Landray MJ, Reith $\mathrm{C}$, et al. The effects of lowering LDL cholesterol with simvastatin plus ezetimibe in patients with chronic kidney disease (Study of Heart and Renal Protection): a randomised placebo-controlled trial. Lancet 2011;377:2181-92.

14. Jafar TH, Stark PC, Schmid CH, et al. Progression of chronic kidney disease: the role of blood pressure control, proteinuria, and angiotensin-converting enzyme inhibition: a patient-level meta-analysis. Ann Intern Med 2003;139:244-52.

15. Toto RD. Treatment of hypertension in chronic kidney disease. Semin Nephrol 2005;25:435-9.

16. KDOQI KDOQI. KDOQI Clinical Practice Guidelines for bone metabolism and disease in children with chronic kidney disease. Secondary KDOQI Clinical Practice Guidelines for bone metabolism and disease in children with chronic kidney disease 2005. http://www. kidney.org/professionals/kdoqi/guidelines_pedbone/guide14.htm

17. Jones-Burton C, Mishra SI, Fink JC, et al. An in-depth review of the evidence linking dietary salt intake and progression of chronic kidney disease. Am J Nephrol 2006;26:268-75.

18. Bronas UG. Cochrane review: in adults with chronic kidney disease regular exercise improves physical fitness, walking capacity, heart rate and blood pressure and some nutritional parameters. Evid Based Nurs 2012;15:95-6.

19. NICE NIfCE. CG73 Chronic kidney disease: full guideline. Secondary CG73 Chronic kidney disease: full guideline 2008. http://guidance.nice.org.uk/CG73/Guidance/pdf/English

20. Levey AS, Bosch JP, Lewis JB, et al. A more accurate method to estimate glomerular filtration rate from serum creatinine: a new prediction equation. Modification of Diet in Renal Disease Study Group. Ann Intern Med 1999;130:461-70. 
21. Eckardt KU, Coresh J, Devuyst O, et al. Evolving importance of kidney disease: from subspecialty to global health burden. Lancet 2013;382:158-69.

22. Manns B, Hemmelgarn B, Tonelli M, et al. Population based screening for chronic kidney disease: cost effectiveness study. BMJ 2010;341: c5869.

23. de Lusignan $\mathrm{S}$, Tomson $\mathrm{C}$, Harris $\mathrm{K}$, et al. Creatinine fluctuation has a greater effect than the formula to estimate glomerular filtration rate on the prevalence of chronic kidney disease. Nephron Clin Pract 2011;117:c213-24.

24. Rule AD, Larson TS, Bergstralh EJ, et al. Using serum creatinine to estimate glomerular filtration rate: accuracy in good health and in chronic kidney disease. Ann Intern Med 2004;141:929-37.

25. Mattix HJ, Hsu CY, Shaykevich S, et al. Use of the albumin creatinine ratio to detect microalbuminuria: implications of sex and race. J Am Soc Nephrol 2002;13:1034-9.

26. NICE NIfCE. BHS \& NICE Hypertension Guideline. Secondary BHS \& NICE Hypertension Guideline 2011. http://guidance.nice.org.uk/ CG127
27. NICE NIfCE. CG66 type 2 diabetes: full guideline. Secondary CG66 type 2 diabetes: full guideline 2008. http://guidance.nice.org.uk/ CG66/Guidance/pdf/English

28. Touze E, Rothwell PM. Heritability of ischaemic stroke in women compared with men: a genetic epidemiological study. Lancet Neurol 2007:6:125-33.

29. Dolan $\mathrm{P}$, Gudex $\mathrm{C}$, Kind $\mathrm{P}$, et al. The time trade-off method: results from a general population study. Health Econ 1996;5:141-54.

30. Dolan P. Modeling valuations for EuroQol health states. Med Care 1997;35:1095-108.

31. Rubin DB, Schenker N. Multiple imputation in health-care databases: an overview and some applications. Stat Med 1991;10:585-98.

32. Briggs A, Clark T, Wolstenholme J, et al. Missing ... presumed at random: cost-analysis of incomplete data. Health Econ 2003;12:377-92.

33. Lattimer N. NICE DSU Technical support Document 14: survival analysis for economic evaluations alongside clinical trials-extrapolation with patient level data. 2011. http://www.nicedsu.org.uk/Other-TSDs (2391674).htm 